This is the peer reviewed version of the following article: "Arias, Francisco J., De Las Heras, Salvador (2019) An estimate for thermal osmotic heat storage using precipitation of common salts. International Journal of Energy Research, 43: 8925-8928." which has been published in final form at [doi: 10.1002/er.4824]. This article may be used for non-commercial purposes in accordance with Wiley Terms and Conditions for Self-Archiving." 


\title{
An Estimate for Thermal Osmotic Heat Storage Using Precipitation of Common Salts
}

\author{
Francisco J. Arias ${ }^{a *}$ and Salvador De Las Heras ${ }^{a}$ \\ Department of Fluid Mechanics, University of Catalonia, \\ ESEIAAT C/ Colom 11, 08222 Barcelona, Spain
}

\begin{abstract}
In this brief note, a first assessment on the possibilities for thermal osmotic storage by harnessing the thermal dependence of the solubility of common salts as an alternative method to sensible heat storage is discussed. In a recent study it was found that such a dependence could be used to run a heat powered cycle (Osmotic Heat Engine). The question raised then, is whether that approach could be suitable for thermal energy storage as well. The attractiveness of such a possibility lies in the capability to store energy for an indefinite period of time without using expensive isolation systems if one considers that osmotic energy only is released when both streams with different salacities are brought together. Utilizing a simplified model, a comparative study with sensible heat storage was performed. It is shown that thermal osmotic storage via thermal precipitation of common salts could be an attractive option when long thermal storage (days) and compactness is desired.
\end{abstract}

Keywords. Sensible heat storage, Osmotic energy.

\section{0}

21

22 ${ }_{24}$ dependence of the solubility featured for common salts 25 for thermal osmotic storage in comparison with tradi${ }_{26}$ tional sensible heat storage. Whereas salinity gradient ${ }_{27}$ based energy storage have been researched in the past 28 in several aspects, [1], nevertheless, as far as the authors 29 know, engineered osmosis has been proposed to store so mostly electrical energy by separating brine and fresh 31 water streams using modified reverse osmosis, and the 32 only osmotic process driven by heat input is related 33 with osmotic heat engines (OHEs) in which heat is 34 applied to the OHE to re-concentrate the draw solution 35 by vaporizing a portion of the water into steam, which 36 would then be condensed to form the deionized working 37 fluid, [2]. Another alternatives involved the chemical 38 precipitation of solutes followed by their re-dissolution 39 or removal of a volatile organic solute Loeb, [4]. More 40 recent, [5], it was proposed that because the thermal ${ }_{41}$ dependence of the solubility of aqueous solutions, it 42 could be possible to harness this feature to convert 43 thermal energy into osmotic energy by separating a 44 given solution into two different streams with different 45 salinities using a process which is pictorially sketched in ${ }_{46}$ Fig. 1. Nevertheless, in that early work, the technique 47 was intended to run powered heat cycles (osmotic heat 48 engines), and the question of whether the method could 49 be attractive for thermal storage in comparison with 50 traditional approach (namely sensible heat) remained as 51 an open question.

52

* Corresponding author:
cisco.javier.arias@upc.edu $\quad$ Tel.: $\quad+\quad+32 \quad 14 \quad 33 \quad 21 \quad 94$; fran-
${ }_{53}$ - Thermal osmotic heat storage vs Sensible 54 heat storage

\section{5}

${ }_{56}$ To begin with, let us consider an upper limit in ${ }_{57}$ the amount of energy which could be stored by the 58 thermal precipitation of common salts in comparison 59 with sensible heat. This amount of energy will depend, 60 of course, on the specific thermal dependence of the ${ }_{61}$ solubility of the given solution which not only may 62 differ greatly between salts but also in the working 63 range of temperatures allowable (see Fig. 2). Thus, for 64 example, the extractable energy from $\mathrm{Na}_{2} \mathrm{SO}_{4}$ could be 65 perhaps around $1 \mathrm{kWh} / \mathrm{m}^{3},[5]$ and limited to a range ${ }_{66}$ of temperature around $300 \mathrm{~K}$ when the strong thermal ${ }_{67}$ dependence of the solubility disappear. On the other 68 hand, another common salt as $\mathrm{KNO}_{3}$ features a stronger 69 thermal dependence of the solubility with an extractable 70 energy up to $5 \mathrm{kWh} / \mathrm{m}^{3}$ or thereabouts and with a 71 broad range of temperature.

72 As regard sensible heat, it is easy to see that sensible 73 heat is able to store much more large amount of energy 74 if one considers that, for example, water with a specific 75 heat capacity $c_{p} \approx 1.16 \mathrm{kWh} /\left(\mathrm{m}^{3} \mathrm{~K}\right)$ and with a temper76 ature difference $\Delta T$ of, say, $20 \mathrm{~K}$, will translate into a 77 higher capability to store energy.

78 Nevertheless, the comparison is not so clear when 79 besides the amount of energy stored it is also taken 80 into consideration the total storing time as well as the 81 compactness of the system as we will see below. 82

83 In order to perform a comparative study in which 84 not only the amount of energy but also the storing 85 time and compactness are considered, let us consider 86 a cylindrical canister as depicted in Fig. 3. In this 87 system, water (which is conspicuous by its high specific 88 heat capacity) is stored at an initial temperature, 


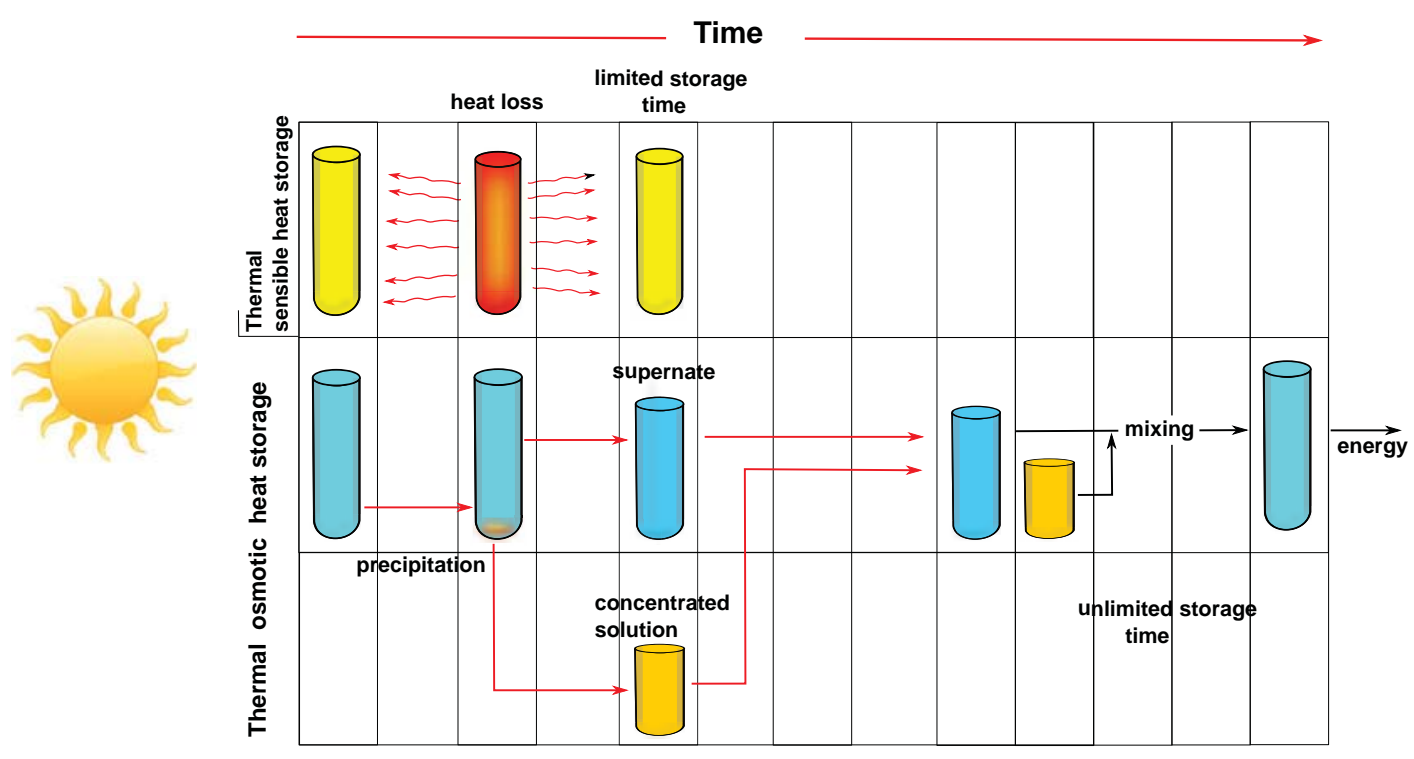

FIG. 1. Comparison between thermal sensible and thermal osmotic heat storage. Although the stored energy by sensible heat could be higher nevertheless the capability for storage is limited because the unavoidable losses with the environment. Contrariwise, osmotic energy can be stored indefinitely and only released when deliberately the two solutions are mixed.

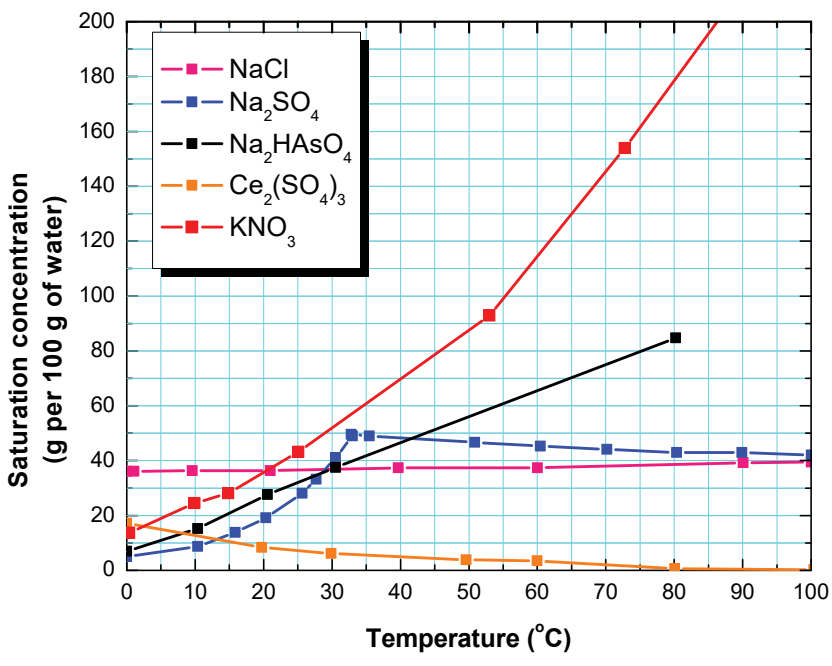

FIG. 2. Solubility vs. temperature for a variety of salts. .

89 say, $T_{i}$ in the canister with radius $r_{1}$ and length $l$ 90 and thus containing a volume of water $\pi r_{1}^{2} l$. This 91 volume is isolated from the environment which is at 92 temperature $T_{o}$ by a second vessel with radius $r_{2}$ and 93 an air-gap in between acting as insulator medium. For 94 a preliminary reckoning, let us neglect axial heat losses 95 in comparison with radial losses which translates into 96 an overestimation of the capability of storing time of ${ }_{97}$ sensible heat and then a conservative safe approximation.

98

99 Under this conditions, the most simple model for the
100 transient thermal conduction between the water and the 101 environment is given by the well known lump expression

$$
\frac{T(t)-T_{o}}{T_{i}-T_{o}}=e^{-\frac{t}{\tau}}
$$

102

where $\tau$ is the so called time constant given by

$$
\tau=\frac{\rho V c_{p}}{A h}
$$

${ }_{103}$ where $\rho$ and $c_{p}$ are the density and heat capacity of the 104 material., respectively; $V$ and $A$ are the volume and the 105 cross-sectional surface area, respectively; and $h$ the heat 106 transfer coefficient. Assuming that the only mechanism 107 for heat transfer between the water and the environment 108 is by thermal conduction, the heat transfer coefficient 109 may be approximated as

$$
\frac{1}{h}=\frac{r_{2}}{\kappa} \ln \frac{r_{2}}{r_{1}}
$$

where $\kappa$ is the thermal conductivity of the air, and $r_{1}$ 111 and $r_{2}$ are the inner and outer radius, respectively. Under 112 our conservative assumption of neglecting the axial heat 113 transfer, we have that $\frac{V}{A} \approx \frac{r_{1}}{2}$ and then by inserting 114 Eq.(3) into Eq.(2) one obtains

$$
\tau=\frac{\rho c_{p} r_{1}^{2}}{2 \kappa}\left[\frac{r_{2}}{r_{1}} \ln \frac{r_{2}}{r_{1}}\right]
$$

115 The sensible energy stored per unit of volume $E_{v}(t)$ at 116 a given time $t$ is calculated from Eq.(1) and yields 


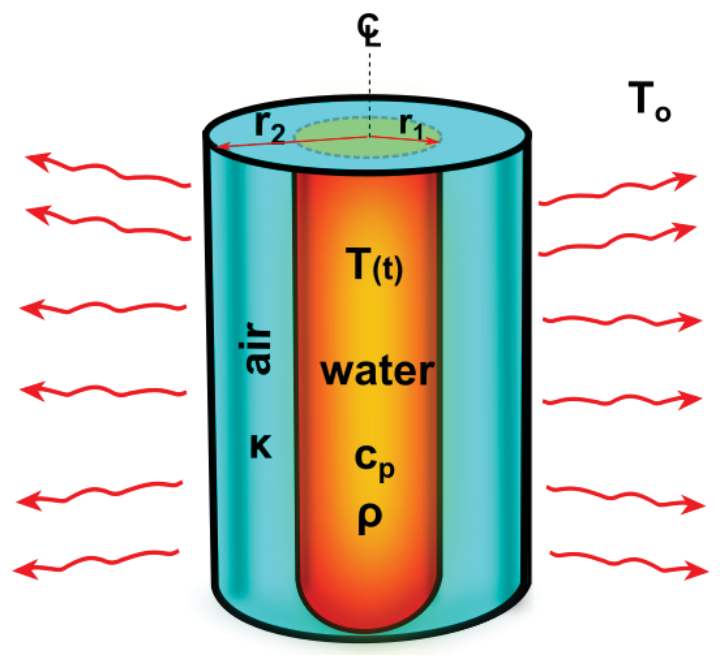

FIG. 3. Physical model for the transient thermal conduction between the water and the environment.

$$
E_{v}(t)=\left(c_{p} \rho \Delta T\right) \cdot e^{-\frac{t}{\tau}}
$$

117 where $\Delta T=T_{i}-T_{o}$ is the initial difference of 118 temperature. Finally, by rearranging terms, we find the 119 storing time as function of the final energy as 120

$$
t=-\tau \ln \left[\frac{E_{v}}{\rho c_{p} \Delta T}\right]
$$

${ }_{121}$ Therefore, thermal osmotic storage could be an attrac122 tive option when the required storable time $t$ is so large 123 that the sensible heat drops below the magnitude of the 124 energy able to be stored indefinitely by osmosis. If one 125 takes this threshold of osmotic energy $E_{\pi} \approx 5 \mathrm{kWh} /\left(\mathrm{m}^{3}\right)$ 126 for a salt featuring a strong thermal dependence with sol${ }_{127}$ ubility as is the case for $\mathrm{KNO}_{3}$ we obtain that for a time 128 larger than

$$
t \approx-\tau \ln \left[\frac{5 k W h / m^{3}}{\rho c_{p} \Delta T}\right]
$$

129 Osmotic thermal storage starts to be an attractive op130 tion

\section{1 - Discussion}

132

133 In order to obtain some idea of the shape of the curves 134 predicted by Eq.(7), we assume some typical values of 135 the parameters using water as thermal fluid and a air as 136 isolator: for water $c_{p}=1.16 \mathrm{Wh} /(\mathrm{kg} \mathrm{K})$; and $\rho=10^{3}{ }_{144}$ the sensible heat in the canister drops just at the limit $137 \mathrm{~kg} / \mathrm{m}^{3}$; for the air $\kappa=0.025 \mathrm{~W} /(\mathrm{m} \mathrm{K})$. The resulting 145 given by osmotic storing because the heat losses with ${ }_{138}$ curves are shown in Fig. 4 and Fig. 5 for a canister 146 the environment. For example, referring to Fig. 4, for 139 with radius $r_{1}=5 \mathrm{~cm}$ and $r_{1}=2 \mathrm{~cm}$ as a function of 147 a canister with $5 \mathrm{~cm}$ and a ration $\frac{r_{2}}{r_{1}}=1.15$, i.e., with

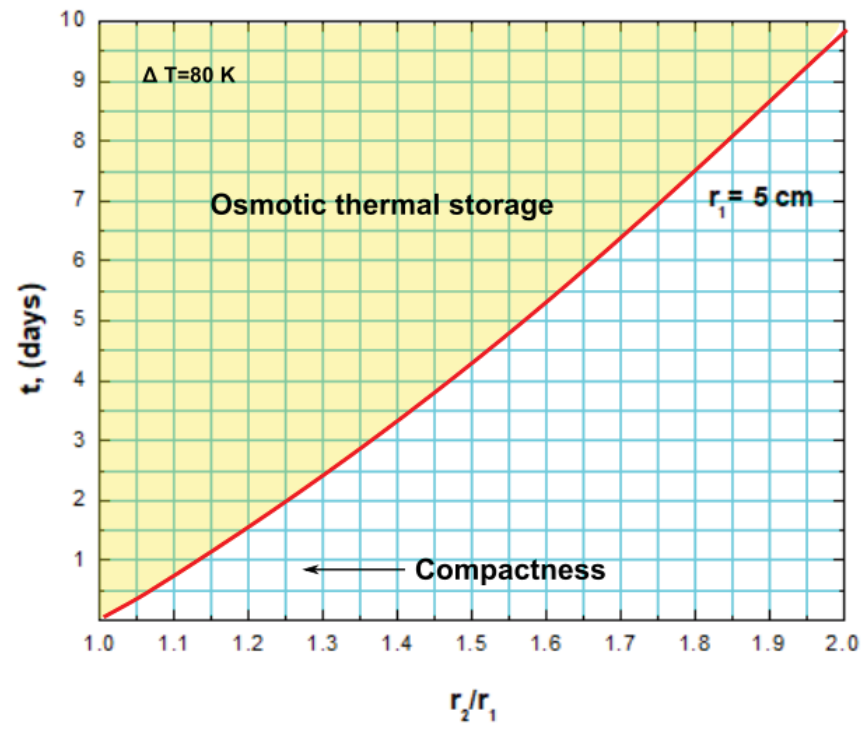

FIG. 4. Thermal storing time as a function of the ratio between the isolation chamber $r_{2}$ and the canister $r_{1}$ as depicted in Fig. 2, and for a canister with radius $r_{1}=5 \mathrm{~cm}$.

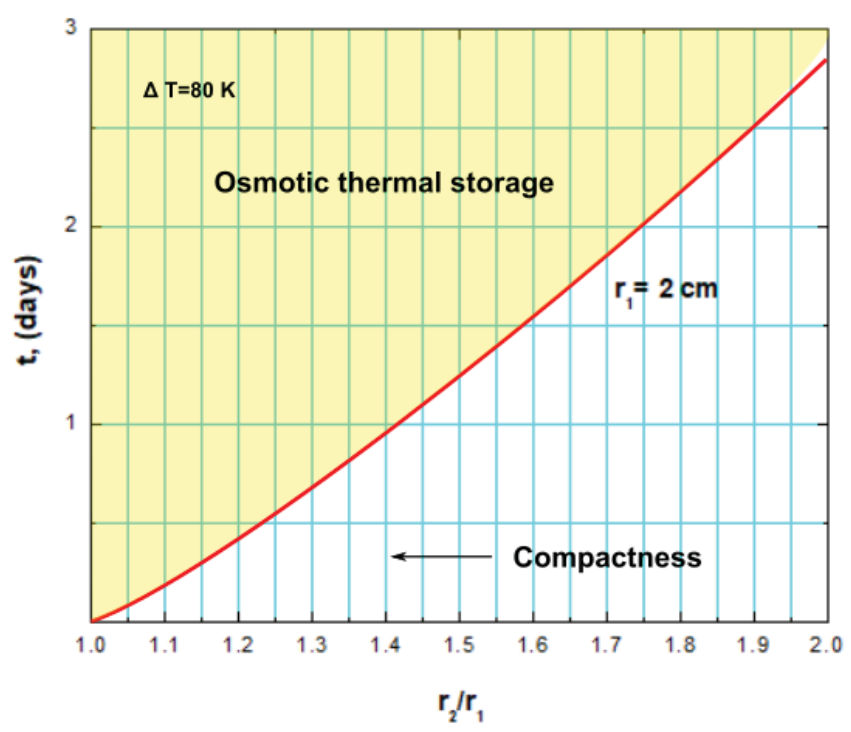

FIG. 5. Thermal storing time as a function of the ratio between the isolation chamber $r_{2}$ and the canister $r_{1}$ as depicted in Fig. 2, and for a canister with radius $r_{1}=2 \mathrm{~cm}$.

140 the ratio between the radius of the isolator $r_{2}$ and the

${ }_{141}$ canister $r_{1}$ (see Fig. 3), $\frac{r_{2}}{r_{1}}$ i.e., the compactness of the 142 system and with an initial $\Delta T=80 \mathrm{~K}$. In those Figures 143 the curve is given the time for a given compactness where

晋

.


148 an air-gap $r_{2}-r_{1}=6.0 \mathrm{~cm}$, the canaster will be only 173 149 able to store energy higher than osmotic up to $\approx 4$ days ${ }_{174}$ 150 or thereabouts, if one wants more time it will necessary 151 using a larger isolator. The compactness can be a limiting 175 152 factor not only because the availability of space but also ${ }_{176}$ 153 in terms of the cost of the overall system.

\section{$154 \quad$ I. SUMMARY OF RESULTS AND \\ 155 CONCLUSIONS}

156 In this note, a scoping study was performed in or157 der to asses the attractiveness of osmotic thermal stor158 age by harnessing the thermal dependence of common 185 159 salts in comparison with sensible heat storage. Utilizing ${ }_{18}$ 160 a simplified geometrical and transient thermal conduc161 tive model it was analyzed under what conditions the 162 approach could be interesting. . It is shown that ther${ }_{163}$ mal osmotic storage via thermal precipitation of common ${ }_{190}$ ${ }_{164}$ salts could be an attractive option when long thermal ${ }_{191}$ 165 storage (days) and compactness is desired. The interest- ${ }_{192}$ 166 ing conclusions derived from this preliminary work are as ${ }_{193}$ 167 follows:

(a) Sensible heat could store a larger amount of thermal energy per volume than that stored by osmosis.

(b) However, if storing time and compactness of the system is considered, osmotic thermal storage could ${ }^{197}$ be an attractive option. (d) Additional R\&D is required in order to arrive at a reliable practical and commercial design

\section{Nomenclature}

$c_{p}=$ heat capacity

$E=$ energy

${ }_{180} h=$ heat transfer coefficient

$181 t=$ time

${ }_{182} T=$ temperature

$r_{1}=$ inner radius

$r_{2}=$ outer radius 185

Greek symbols

${ }_{187} \kappa=$ thermal conductivity

$188=$ density

$\tau=$ time constant

\section{Acknowledgements}

This research was supported by the Spanish Ministry 194 of Economy and Competitiveness under fellowship grant 195 Ramon y Cajal: RYC-2013-13459.

\section{REFERENCES}

198 [1] Bharadwaj D; Struchtrup H. 2017. Large scale energy stor- 209 199 age using multistage osmotic processes: approaching high 210 200 efficiency and energy density. Sustainable Energy Fuels, 211 $201 \quad 2017,1,599-614$

202 [2] McGinnis R.L; McCutcheon J.R; Elimelech M. 2007. 213 203 A novel ammonia-carbon dioxide osmotic heat engine 214 204 for power generation. Journal of Membrane Science 305215 $205 \quad$ (2007) 13-19

206 [3] Loeb S. 1975. Method and apparatus for generating power 217 207 utilizing pressure retarded- osmosis, US Patent 3,906,250, 218 208 Ben-Gurion University of the Negev Research and Devel- opment Authority, Beersheba, Israel, US.

4] Reali M. 1980. Closed cycle osmotic power-plants for electric-power production, Energy 5 (4). 325-329.

[5] Arias F.J. 2018. On osmotic heat engines driven by thermal precipitation-dissolution of saturated aqueous solutions. International Journal of Thermal Sciences. 133, p.p. 151-161

[6] Lin S; Strauba A.P; Elimelech M. 2014. Thermodynamic limits of extractable energy by pressure retarded osmosis. 8 Eenergy Environ. Sci. 2014. 7. 2706. 